\title{
The Blazhko effect of RR Lyrae in 2003-2004
}

\author{
K. Kolenberg ${ }^{1,2}$, H. A. Smith ${ }^{3}$, K. D. Gazeas ${ }^{4}$, A. Elmasl1 ${ }^{5}$, M. Breger ${ }^{1}$, E. Guggenberger ${ }^{1}$, P. Van Cauteren ${ }^{6}$, \\ P. Lampens ${ }^{7}$, P. Reegen ${ }^{1}$, P. G. Niarchos ${ }^{4}$, B. Albayrak ${ }^{5}$, S. O. Selam ${ }^{5}$, İ. Özavc1 ${ }^{5}$, and O. Aksu ${ }^{5}$ \\ 1 Institute for Astronomy, University of Vienna, Türkenschanzstrasse 17, 1180 Vienna, Austria \\ e-mail: kolenberg@astro.univie.ac.at \\ 2 Instituut voor Sterrenkunde, Katholieke Universiteit Leuven, Celestijnenlaan 200B, 3001 Leuven, Belgium \\ 3 Department of Physics and Astronomy, Michigan State University, East Lansing, MI 48824-2320, United States \\ 4 Department of Astrophysics, Astronomy and Mechanics, University of Athens, Panepistimiopolis, 15784 Zografos, Athens, Greece \\ 5 Ankara University, Faculty of Science, Dept. of Astronomy and Space Sciences, 06100 Tandoğan, Ankara, Türkiye \\ 6 Beersel Hills Observatory, Belgium \\ 7 Royal Observatory, Ringlaan 3, 1180 Brussel, Belgium
}

Received 25 October 2005 / Accepted 22 June 2006

\section{ABSTRACT}

\begin{abstract}
Aims. Extensive photometry of RR Lyr was obtained over a 421-day interval in 2003-2004, covering more than 10 Blazhko cycles in a multisite campaign. The length and density of this data set allow for a detailed analysis.

Methods. We used Fourier techniques to study RR Lyr's behavior over the pulsation and the Blazhko cycle. We propose a twofrequency model for decomposing the frequency spectrum.

Results. The light variations were fitted with the main radial frequency, its harmonics up to 11th order, and the detected triplet frequencies. No significant quintuplet components were found in the frequency spectrum. Given the total time span of the measurements, we can now unambiguously conclude that the Blazhko period has become notably shorter than the previously known value of 40.8 days, whereas the main pulsation period remained roughly the same. Changes in the modulation period have been reported for other well-studied Blazhko variables. They challenge the explanations for the Blazhko effect which link the modulation period directly to the rotation period. The new photometry reveals an interval in the pulsation cycle of RR Lyr during which the star's intensity barely changes over the Blazhko cycle. This interval occurs during the infalling motion and between the supposed phases of the early and the main shock. The data also permit a more detailed study of the light curve shape at different phases in the Blazhko period through Fourier parameters.
\end{abstract}

Key words. stars: oscillations - stars: individual: RR Lyr

\section{Introduction}

Although the time elapsed since the discovery of the Blazhko effect among the well-known class of RR Lyrae stars approaches one century, the phenomenon still remains a mystery. The Blazhko effect is a periodic amplitude and/or phase modulation shown by some $20-30 \%$ of the galactic RRab stars (fundamental mode pulsators - see Szeidl 1988; Moskalik \& Poretti 2003; Kovács 2005). These stars, with pulsation periods of about half a day, have light curves that are modulated on timescales of typically tens to hundreds of days. Blazhko (1907) was the first to report this phenomenon in RW Dra. In the LMC the occurrence rate of Blazhko stars is considerably lower (about 12\%; Alcock et al. 2003). RRc stars (first overtone pulsators) show the Blazhko effect even less frequently (less than 5\%; Alcock et al. 2000).

\subsection{Explanations for the Blazhko effect}

Nowadays, two classes of models are usually put forward as possible explanations for the Blazhko effect. Though RR Lyrae stars have for a long time been considered as the prototypes of purely radially pulsating stars, both currently proposed models involve the presence of nonradial oscillations.

In the resonance models nonradial modes of low degree $\ell$ can be nonlinearly excited through a resonance with the main radial mode (Cox 1993; Van Hoolst et al. 1998; Nowakowski \& Dziembowski 2001). The probability of excitation is the highest for dipole $(\ell=1)$ modes (Van Hoolst et al. 1998). The additional nonradial pulsation components lead to a triplet structure in the frequency spectrum of Blazhko variables. In the model proposed by Nowakowski \& Dziembowski (2001) the excitation of a rotationally split $m= \pm 1$ pair can cause significant amplitude and phase modulation. In this model the modulation period is determined by the rotation rate, but also by the Brunt-Väisälä frequency in the deepest part of the radiative interior. As a consequence, in these models the ratio between rotation and modulation period is not expected to be an integer number.

The magnetic models proposed for the Blazhko effect are similar to the oblique pulsator model for roAp stars (Kurtz 1982), in which the magnetic axis of the star is inclined to its rotation axis. The magnetic field gives rise to an additional quadrupole component $(\ell=2)$ whose symmetry axis coincides with the magnetic axis. As the star rotates, we observe the pulsation components under different angles, which causes the 
observed amplitude modulation. The additional dipole components lead to a quintuplet structure in the frequency spectrum of a Blazhko star, but Shibahashi \& Takata (1995) also show that the quintuplet looks almost like a triplet for certain geometrical configurations. Depending on which of the (quintuplet) side components we observe, the Blazhko period is supposed to be equal to the rotation period or half the rotation period.

Based on the evidence that some Blazhko stars also display a long period cycle of the order of some years besides the radial pulsation and the Blazhko cycle (about 4 years for RR Lyr, see Detre \& Szeidl 1973), several authors proposed the existence of a magnetic cycle. A magnetic field of the order of $1 \mathrm{kGauss}$ would be required in the model of Shibahashi \& Takata (1995) to reproduce the observed amplitude modulation. Babcock (1958) and Romanov et al. (1994) report on the detection of a variable magnetic field in RR Lyr with a strength up to $1.5 \mathrm{kGauss}$, but so far, these observations have been seriously questioned. More recently, the presence of a magnetic field of the required strength was rejected on the basis of observations carried out over 4 consecutive years by Chadid et al. (2004), hence allowing for detection of variations over the "4-year cycle".

The closest match between Blazhko stars and non-Blazhko stars occurs at the Blazhko phase which corresponds to the largest amplitude (see diagrams in Teays 1993). These results seem to indicate that the Blazhko effect is connected with a mechanism which tends to suppress the normal pulsational features of the star (Szeidl 1976). ă An interesting study in this respect was carried out by Jurcsik et al. (2002a), through a comparison of the light and radial velocity amplitudes as well as the light curve shapes (using Fourier parameters) between Blazhko stars and so-called "normal", non-modulated, RR Lyrae stars. They concluded that, for Blazhko stars with large amplitude modulation, there is no Blazhko phase that seems to be wellmatched with that of a non-modulated RRab star.

\subsection{Extensive photometric observations}

The Blazhko effect has been the frequent subject of photographic and photometric studies, e.g., the systematic investigations carried out at Konkoly Observatory in Budapest, Hungary (Szeidl \& Kolláth 2000, and references therein). Traditionally the phenomenon was studied by means of $\mathrm{O}-\mathrm{C}$ analysis. Therefore the observations were in general strongly biased towards the ascending branch and maximum phase of the primary light curve, a sampling pernicious for Fourier analyses (see also Kovács 2001). Photometric data sets including also other parts of the light curve allowed for a rigorous frequency analysis, e.g., Borkowski (1980), Kovács (1995), Szeidl \& Kolláth (2000), Smith et al. (2003), Jurcsik et al. (2005).

Over the past decade, the outcome of large-scale studies such as microlensing surveys (e.g., OGLE, see Moskalik \& Poretti 2003; MACHO, see Alcock et al. 2000, 2003) has yielded a much better view of the general phenomenology of the Blazhko effect, since a large number of previously unknown Blazhko stars were detected and analyzed. Even though such extensive multi-target surveys have revolutionized the subject, long-term multi-site campaigns dedicated to particular Blazhko stars remain of great scientific value. Only observations including complete light curves of the target stars can reveal long-term changes in the characteristics of the Blazhko effect (such as amplitudes, phases, and periods). As such they can give crucial information for deciding among the different hypotheses for the Blazhko effect.
At the end of 2003 an international collaboration (the Blazhko Project, see also Kolenberg 2005) was set up, focused on obtaining a better understanding of the Blazhko effect through extensive observations of a sample of critically selected Blazhko stars. Photometric data gathered over several Blazhko cycles ensure the required frequency resolution. At the same time, spectroscopic data collected at well-chosen time intervals over the Blazhko cycles of the targets can reveal decisive information on the pulsation modes occurring in these stars. For details on the project and for an updated status and outcome of the ongoing campaigns we refer to the dedicated website: http://www . univie.ac.at/tops/blazhko/

One of the first main targets of the Blazhko Project was RR Lyr, the brightest and probably the best studied RR Lyrae star. From October 2003 onwards photometric data of this star were obtained with the 0.6-m telescope at Michigan State University, and during the whole observational season of 2004 six telescopes (0.13-m up to $0.8-\mathrm{m})$ acquired photometric data.

In this paper we present the results of the photometric campaign on RR Lyr in 2004. In Sect. 2 we present the observations and their derived phasing. The data analysis and its results are described in Sect. 3, with special emphasis on new findings. Sect. 4 is dedicated to a discussion of the results, along with the present status of the interpretations of the Blazhko effect. Finally, some concluding remarks are given in Sect. 5.

\section{Observations}

Our photometric observations were carried out with six different telescopes in the northern hemisphere. Four of the telescopes were equipped with CCD cameras, and two used photomultipliers to obtain comparative photometry of RR Lyr. We will discuss the combined Johnson $V$ band photometry gathered at all the observatories.

The time spanned by the 2003 data is 35.04 days (from HJD 2452915.53 to HJD 2452950.57 ); the time spanned by the 2004 data alone is 193.99 days (from HJD 2453142.62 till HJD 2453336.61 ), i.e., almost 5 complete Blazhko cycles. The total time spanned by the 2003-2004 measurements is 421.08 days, covering more than 10 Blazhko cycles. Table 1 shows a journal of the observations. Table 2 displays the observational details.

Small zero-point shifts were carried out to combine the data gathered from different instruments. The resulting differential magnitudes are given with respect to $\mathrm{BD}+42$ 3334. The accuracy of an individual differential measurement is typically \pm 0.01-0.02 mag. Castelaz et al. (1991) list $V=10.19$ and $B-V=0.25$ for BD +423334 in their table with photometry of astrometric reference stars.

From the 2004 measurements we determined the following ephemerides of maximum pulsation light and maximum pulsation amplitude:

$\operatorname{HJD}\left(T_{\max }\right)=2453250.576+0.566839 \times E_{\text {pulsation }}$

$\operatorname{HJD}\left(T_{\mathrm{B} 1 \max }\right)=2453250.576+38.8 \times E_{\text {Blazhko }}$.

Note that the Blazhko period in the new ephemeris has changed considerably compared to previous ephemerides dating back to 1996 as given by Chadid et al. (1997). We will discuss this in more detail in Sects. 3.1 and 4.3. 
Table 1. Log of the photometric Johnson $V$ band observations of RR Lyr.

\begin{tabular}{lcccccl}
\hline \hline Observatory & $N$ Data & $N$ Nights & $N$ Hours & HJD(begin) & HJD(end) $^{*}$ & Observers \\
\hline Michigan State University & $700^{* *}$ & $7^{* *}$ & $11.05^{* *}$ & $2915.5289^{* *}$ & $2950.5715^{* *}$ & HAS \\
Michigan State University & 7492 & 55 & 141.96 & 3142.6229 & 3322.5803 & HAS \\
Athens University Observatory & 4810 & 7 & 49.91 & 3213.3158 & 3278.4990 & KDG, PGN \\
APT Arizona & 457 & 15 & 45.57 & 3184.8050 & 3336.6100 & robotic \\
Ankara University Observatory & 649 & 6 & 34.72 & 3224.2939 & 3268.4783 & AE, BA, SOS, IO, OA \\
Beersel Hills Observatory & 232 & 5 & 9.25 & 3305.2902 & 3319.3134 & PVC, PL \\
Vienna Observatory & 362 & 3 & 7.83 & 3253.3476 & 3253.5062 & KK \\
\hline
\end{tabular}

${ }^{*} \mathrm{HJD}(-2450000) ;{ }^{* *} 2003$ data.

Table 2. Observational details of the data set described in this paper.

\begin{tabular}{|c|c|c|c|c|c|}
\hline$\overline{\text { Observatory }}$ & Telescope & Equipment & Field of view & Pixel scale & $\overline{\text { Exp. interval (s) }}$ \\
\hline Michigan State University $^{(1)}$ & $0.6-\mathrm{m}$ & Apogee Ap47p CCD & $10 \times 10^{\prime}$ & $1.1^{\prime \prime} / \mathrm{pix}$ & $60-90$ \\
\hline Athens University Observatory ${ }^{(1)}$ & $0.4-\mathrm{m}$ & SBIG ST8 CCD & $15 \times 22.5^{\prime}$ & $2.6^{\prime \prime} / \mathrm{pix}$ & $30-40$ \\
\hline APT Arizona ${ }^{(2)}$ & $0.7-\mathrm{m}$ & EMI-9124QB PMT & - & - & 40 \\
\hline Ankara University Observatory ${ }^{(2)}$ & $0.3-\mathrm{m}$ & SSP-5A PMT & - & - & 40 \\
\hline Beersel Hills Observatory $^{(3)}$ & $0.13-\mathrm{m}$ & ST10XME CCD & $43.8 \times 29.4^{\prime}$ & $1.2^{\prime \prime} / \mathrm{pix}$ & $50-130$ \\
\hline Beersel Hills Observatory $^{(3)}$ & $0.25-\mathrm{m}$ & ST10XME CCD & $33.9^{\prime} \times 22.9^{\prime}$ & $0.9^{\prime \prime} / \mathrm{pix}$ & $50-130$ \\
\hline Vienna Observatory $^{(1)}$ & $0.8-\mathrm{m}$ & Apogee Ap9e CCD & $10^{\prime} \times 14^{\prime}$ & $0.3^{\prime \prime} / \mathrm{pix}$ & $60-90$ \\
\hline
\end{tabular}

(1) Comparison star: BD +42 3334, check star: BD +42 3339 .

(2) Comparison star: HD182755 = BD+42 3331, check star: BD +42 3334.

(3) Comparison star: GSC $314268=\mathrm{SAO} 48408$, check star: GSC.

\section{Data analysis}

\subsection{Fourier analysis}

The frequency analyses were performed with Period04 (Lenz \& Breger 2005), a package applying single-frequency power spectrum and simultaneous multi-frequency sine-wave fitting.

The fact that data were obtained from observatories at different longitudes improved the spectral window function in the sense that one-day aliases are much weaker (see Fig. 1). Given the large time gap between the 2003 and 2004 data and the relatively small number of data available from 2003 (700 points), we started with a thorough analysis of the 2004 data (14 002 points). In this way, we could also verify whether the star's behavior remained stable from one year to the next.

Extensive light and radial velocity curves of RR Lyrae Blazhko stars exhibit in their frequency spectra either a doublet structure or an equally-spaced triplet structure around the main pulsation frequency $f_{0}$ and its harmonics $k f_{0}$, with a small frequency separation corresponding to the Blazhko frequency $f_{\mathrm{B}}$ (see Kovacs 2001; Alcock et al. 2000, 2003). Extensive light curves for RR Lyr have been published by several authors (e.g. Balasz \& Detre 1943; Szeidl et al. 1997), and it has been reported that Fourier analyses of the light curves display a triplet structure (e.g. Smith et al. 2003). Radial velocity curves for RR Lyr have also been published (e.g. Struve \& Blaauw 1948; and Preston et al. 1965), with Chadid et al. (1999) reporting the existence of a triplet structure in the radial velocity variations.

Fourier analyses were carried out on the photometric data. When searching for frequencies through successive prewhitening, we first find the main frequency $f_{0}$ and its first two harmonics, $2 f_{0}$ and $3 f_{0}$. Then the right side peak of the triplet at the main frequency appears, $f_{0}+f_{\mathrm{B}}$, followed by $4 f_{0}$, and right side peaks of higher order. The first left side peak to appear is one of higher order, $3 f_{0}-f_{\mathrm{B}}$, closely followed by $2 f_{0}-f_{\mathrm{B}}$. The left side peak of the main frequency, $f_{0}-f_{\mathrm{B}}$ has a low but significant amplitude. Figure 2 shows the results of successive prewhitening

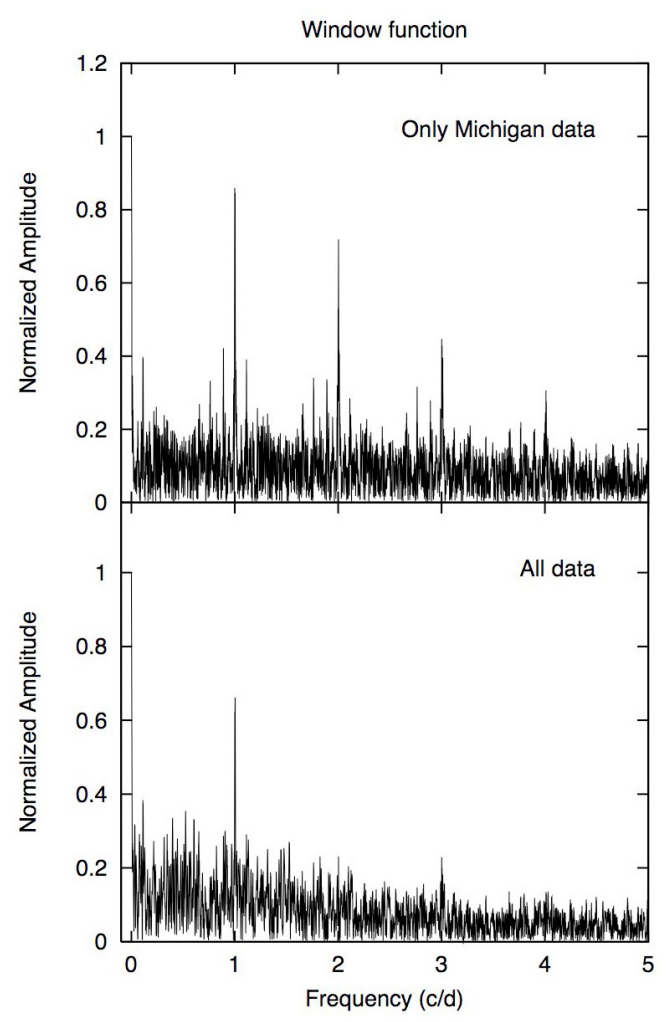

Fig. 1. Comparison of the spectral windows for the Michigan data only (the largest data set, top), and the combined data, including the other observatories (bottom).

in the Fourier spectrum of the data set. For comparison with photometric results on previous data sets of RR Lyr, we present the results expressed in magnitudes. Table 3 list the results of a 


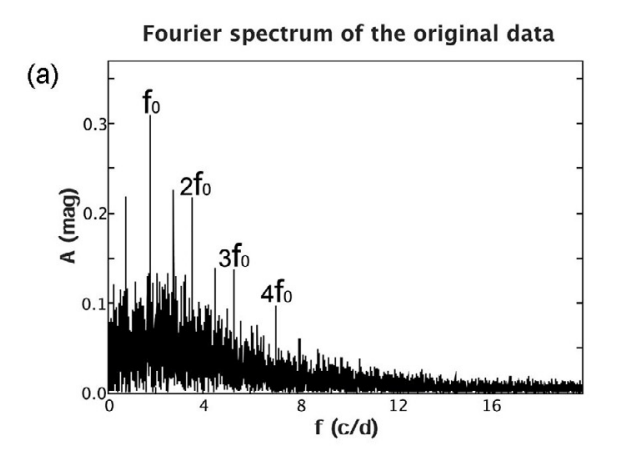

Right side peak after subtraction of fo and its harmonics

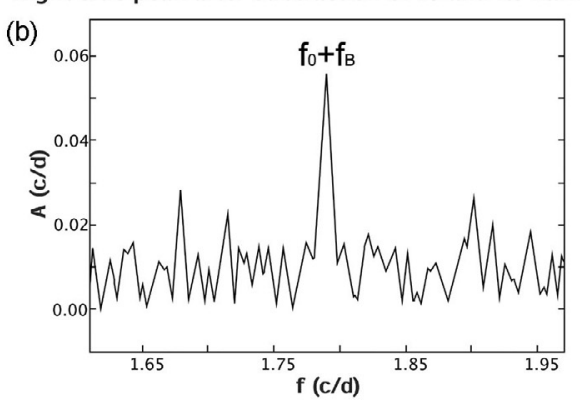

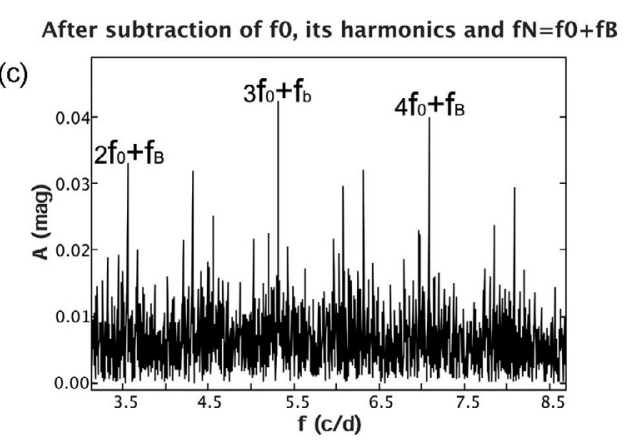

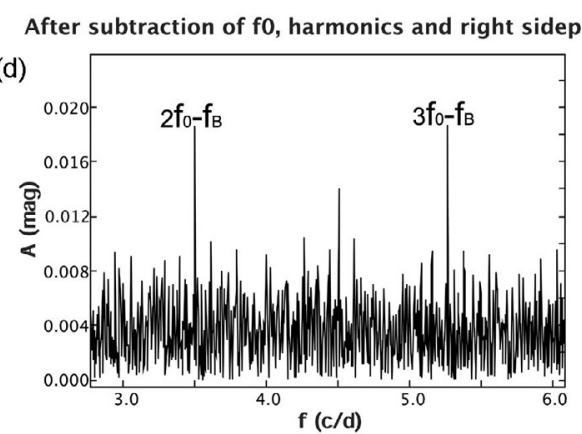

Fig. 2. Fourier spectra (amplitude versus frequency) at different stages of the period search process. Panel a) shows the Fourier spectrum of the original data. The labeled peaks are the main frequency $f_{0}$ and its harmonics. Panel b) zooms into the region of the right side peak frequency $f_{\mathrm{N}}=f_{0}+f_{\mathrm{B}}$, the first frequency appearing after $f_{0}$ and its harmonics. Subsequently, panel c) displays the higher order right side peak frequencies. Finally, panel d) shows the first left side peak frequencies to appear in the Fourier spectrum after successive prewhitening. In all panels the unlabeled peaks of considerable amplitudes are 1-day aliases of real frequencies.

multifrequency fit to the combined set of 2004 data, according to the formula (see also Kovács 1995):

$$
\begin{aligned}
f(t)= & A_{0}+\sum_{k=1}^{n}\left[A_{k} \sin \left(2 \pi k f_{0} t+\phi_{k}\right)\right. \\
& +A_{k+} \sin \left(2 \pi\left(k f_{0}+f_{\mathrm{B}}\right) t+\phi_{k+}\right) \\
& \left.+A_{k-} \sin \left(2 \pi\left(k f_{0}-f_{\mathrm{B}}\right) t+\phi_{k-}\right)\right] \\
& +B_{0} \sin \left(2 \pi\left(f_{\mathrm{B}} t+\phi_{\mathrm{B}}\right)\right),
\end{aligned}
$$

where we calculated amplitudes and phases up to order $n=11$, since the amplitudes of the higher-order harmonics went below the significance level. In the fit, $f_{0}$ is the main pulsation frequency and $f_{\mathrm{B}}$ is the Blazhko frequency. We calculated optimum values for the frequencies, their amplitudes and phases by minimizing the residuals. Errors on the values for the frequencies, amplitudes and phases were calculated following Breger et al. (1999) and Montgomery \& O’Donoghue (1999). Since correlated noise may lead to an underestimation of these errors, we multiplied them by a factor 2 in order to get realistic values, following the conclusions of Handler et al. (2000) and Jerzykiewicz et al. (2005). We compared the obtained error values with those yielded by extensive Monte Carlo simulations, and concluded they are of the same order of magnitude.

From this data set and with the fit according to Eq. (1), we obtained $f_{0}=1.7641704 \pm 0.0000048 \mathrm{c} / \mathrm{d}$ for the main radial frequency, corresponding to a period $P_{0}=0.5668386 \pm$ $0.0000016 \mathrm{~d}$. The right side peak at the main frequency is at $f_{\mathrm{N}}=f_{0}+f_{\mathrm{B}}=1.7899187 \pm 0.000026 \mathrm{c} / \mathrm{d}$. For the Blazhko frequency we then get a value of $f_{\mathrm{B}}=0.025748 \pm 0.000026 \mathrm{c} / \mathrm{d}$, as the difference between the main peak and the side peak in the triplet structure. The Blazhko period obtained in this way from this data set is thus $P_{\mathrm{B}}=38.84 \pm 0.06 \mathrm{~d}$. This period is considerably shorter than the previously assumed value of 40.8 days. The change in the Blazhko period will be discussed in Sect. 4.3.
In our analyses we adopt a significance criterion of amplitude signal-to-noise ratios of 4.0 for non-combination frequencies and 3.5 for combination frequencies, following Breger et al. (1993). The values in Table 3 displayed in italics correspond to combination frequencies not exceeding a signal-to-noise level of 3.5. Although the model proposed in Eq. (3) contains a term varying with the modulation frequency $f_{\mathrm{B}}$, the data set does not reveal any significant variation with this frequency.

Though there is some remaining signal in the residuals of the obtained fit to the data (see Fig. 8), no other frequencies were found in the data with a signal exceeding the applied signal-tonoise ratio threshold. From these data we observed that there is no exact repetition of the light curve shape from one Blazhko cycle to the next. This called for caution in directly including the RR Lyr photometry (700 data, $11.05 \mathrm{~h}$ ) from 2003 in the fit, since small phase shifts or changes of the zero point would deteriorate the obtained fit.

RR Lyr is known to have a long-term cycle of about 4 years, at the end of which the strength of the amplitude and phase modulation suddenly decreases and then recovers (Detre \& Szeidl 1973). This event is also observed to be accompanied by a shift in the Blazhko phasing. Chadid \& Gillet (1997) noticed that the end of 1994 corresponded to the beginning of a new "4year cycle". If the cycle indeed still has a period ranging from 3.8 to 4.8 years as reported by Detre \& Szeidl (1973), a new cycle would have been likely to start between mid 2002 and mid 2004, i.e., it might have restarted within the time span of our observations. At previous transitions a shift in the Blazhko phase has been observed (Detre \& Szeidl 1973). We included the 2003 data in the solution only after obtaining an optimal fit for the 2004 data, which show no indication of a large shift in the Blazhko period. For this fit the obtained frequency values, their amplitudes and phases remain the same within the given error bars. Within the observational errors, they agree well with the fit 
Table 3. Amplitudes and phases (in degrees) of the pulsation and modulation frequency components of RR Lyr from the 2004 photometric data. The values displayed in italics correspond to combination frequencies not exceeding a signal-to-noise level of 3.5. For non-combination frequencies the signal-to-noise ratio threshold is 4.0. The residuals of the fit are at $0.021 \mathrm{mag}$, which is about the noise level of the data set.

\begin{tabular}{|c|c|c|c|c|c|}
\hline \multirow[b]{2}{*}{$\begin{array}{l}\text { Modulation } \\
\text { model }\end{array}$} & \multicolumn{2}{|c|}{$\overline{f f[\mathrm{c} / \mathrm{d}]}$} & \multirow{2}{*}{$\begin{array}{l}A[\mathrm{mag}] \\
\Delta A=0.00052\end{array}$} & \multirow[t]{2}{*}{ 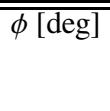 } & \multirow[t]{2}{*}{$\overline{\Delta \phi}[\mathrm{deg}]$} \\
\hline & $\begin{array}{l}\text { Two-frequency } \\
\text { model }\end{array}$ & Value & & & \\
\hline & $f_{0}$ & $1.7641704 \pm 0.0000048$ & 0.29471 & 25.71 & 0.32 \\
\hline & $2 f_{0}$ & 3.5283407 & 0.13340 & 189.9 & 1.4 \\
\hline & $3 f_{0}$ & 5.2925111 & 0.08794 & 12.6 & 2.14 \\
\hline & $4 f_{0}$ & 7.0566814 & 0.04880 & 200.62 & 3.8 \\
\hline & $5 f_{0}$ & 8.8208517 & 0.02497 & 33.4 & 7.4 \\
\hline & $6 f_{0}$ & 10.5850221 & 0.01417 & 234.8 & 13.2 \\
\hline & $7 f_{0}$ & 12.3491924 & 0.00806 & 77.7 & 23.2 \\
\hline & $8 f_{0}$ & 14.1133628 & 0.00596 & 283.6 & 31.4 \\
\hline & $9 f_{0}$ & 15.8775331 & 0.00474 & 112.0 & 39.4 \\
\hline & $10 f_{0}$ & 17.6417035 & 0.00387 & 287.7 & 48.4 \\
\hline & $11 f_{0}$ & 19.4058738 & 0.00355 & 125.5 & 52.8 \\
\hline$f_{0}+f_{\mathrm{B}}$ & $=f_{\mathrm{N}}$ & $1.7899187 \pm 0.000026$ & 0.05640 & 135.8 & 3.4 \\
\hline$f_{0}-f_{\mathrm{B}}$ & $=2 f_{0}-f_{\mathrm{N}}$ & 1.7384220 & 0.00959 & 147.8 & 19.6 \\
\hline $2 f_{0}+f_{\mathrm{B}}$ & $=f_{0}+f_{\mathrm{N}}$ & 3.5540891 & 0.03068 & 290.9 & 6.2 \\
\hline $2 f_{0}-f_{\mathrm{B}}$ & $=3 f_{0}-f_{\mathrm{N}}$ & 3.5025923 & 0.01427 & 294.6 & 13.2 \\
\hline $3 f_{0}+f_{\mathrm{B}}$ & $=2 f_{0}+f_{\mathrm{N}}$ & 5.3182594 & 0.03371 & 87.5 & 5.6 \\
\hline $3 f_{0}-f_{\mathrm{B}}$ & $=4 f_{0}-f_{\mathrm{N}}$ & 5.2667627 & 0.01462 & 131.2 & 12.8 \\
\hline $4 f_{0}+f_{\mathrm{B}}$ & $=3 f_{0}+f_{\mathrm{N}}$ & 7.0824298 & 0.03130 & 260.0 & 6.0 \\
\hline $4 f_{0}-f_{\mathrm{B}}$ & $=5 f_{0}-f_{\mathrm{N}}$ & 7.0309330 & 0.01048 & 320.5 & 17.8 \\
\hline $5 f_{0}+f_{\mathrm{B}}$ & $=4 f_{0}+f_{\mathrm{N}}$ & 8.8466001 & 0.02200 & 85.0 & 8.4 \\
\hline $5 f_{0}-f_{\mathrm{B}}$ & $=6 f_{0}-f_{\mathrm{N}}$ & 8.7951034 & 0.00496 & 187.8 & 37.8 \\
\hline $6 f_{0}+f_{\mathrm{B}}$ & $=5 f_{0}+f_{\mathrm{N}}$ & 10.6107704 & 0.01327 & 252.1 & 14.2 \\
\hline $6 f_{0}-f_{\mathrm{B}}$ & $=7 f_{0}-f_{\mathrm{N}}$ & 10.5592737 & 0.00285 & 15.9 & 65.6 \\
\hline $7 f_{0}+f_{\mathrm{B}}$ & $=6 f_{0}+f_{\mathrm{N}}$ & 12.3749408 & 0.00978 & 71.4 & 919.2 \\
\hline $7 f_{0}-f_{\mathrm{B}}$ & $=8 f_{0}-f_{\mathrm{N}}$ & 12.3234441 & 0.00393 & 176.6 & 47.6 \\
\hline $8 f_{0}+f_{\mathrm{B}}$ & $=7 f_{0}+f_{\mathrm{N}}$ & 14.1391111 & 0.00637 & 248.5 & 29.4 \\
\hline $8 f_{0}-f_{\mathrm{B}}$ & $=9 f_{0}-f_{\mathrm{N}}$ & 14.0876144 & 0.00302 & 44.0 & 62.0 \\
\hline $9 f_{0}+f_{\mathrm{B}}$ & $=8 f_{0}+f_{\mathrm{N}}$ & 15.9032815 & 0.00393 & 80.1 & 47.6 \\
\hline $9 f_{0}-f_{\mathrm{B}}$ & $=10 f_{0}-f_{\mathrm{N}}$ & 15.8517848 & 0.00218 & 225.4 & 85.8 \\
\hline $10 f_{0}+f_{\mathrm{B}}$ & $=9 f_{0}+f_{\mathrm{N}}$ & 17.6674518 & 0.00193 & 289.0 & 97.0 \\
\hline $10 f_{0}-f_{\mathrm{B}}$ & $=11 f_{0}-f_{\mathrm{N}}$ & 17.6159551 & 0.00309 & 37.8 & 30.3 \\
\hline $11 f_{0}+f_{\mathrm{B}}$ & $=10 f_{0}+f_{\mathrm{N}}$ & 19.4316222 & 0.00033 & 48.1 & 567.2 \\
\hline $11 f_{0}-f_{\mathrm{B}}$ & $=12 f_{0}-f_{\mathrm{N}}$ & 19.3801255 & 0.00216 & 214.6 & 86.6 \\
\hline$f_{\mathrm{B}}$ & $=f_{\mathrm{N}}-f_{0}$ & 0.0257483 & 0.00166 & 154.3 & 112.6 \\
\hline
\end{tabular}

obtained from the 2004 data. This implies that there is no clear indication for the beginning of a new "4-year cycle" between November, 2003 and March, 2004. It rather seems that all data have been obtained within the same "4-year cycle" of RR Lyr.

\subsection{A two-frequency model for decomposing the frequency spectrum}

Equation (3) gives the commonly used frequency decomposition for Blazhko stars, which we refer to as the "modulation model". This decomposition explicitly contains the modulation or Blazhko frequency, which may result from a periodic change of our view upon the star's pulsation, or from a beating between two close frequencies (see Sect. 1.1).

Another mathematically equivalent way of decomposing the frequencies starts from the simple assumption that in reality there are only two modes present in the star, producing the observed frequency spectrum (frequency-amplitude diagram). On the one hand, there is the main radial mode, which in the case of a fundamental mode RRab pulsator, has a large amplitude and hence behaves nonlinearly. We see the harmonics of this frequency up to a high order (up to order 11 in this case). On the other hand, we observe a frequency $f_{\mathrm{N}}$, close to the main frequency, related to an additional mode. Because of its frequency value (too close to a radial mode frequency to be radial itself), this mode must be nonradial and, since it has a small amplitude, it can be considered as behaving in a linear fashion, at least in the zone where most of the pulsation takes place. We note that a model of nonlinear mode superposition was already proposed by Borkowski (1980) to explain the Blazhko effect in the star AR Her as a result of double mode pulsation. Balász \& Detre (1943) also noted that the light variations of RR Lyr can most easily be described by a superposition of two pulsations with frequencies $f_{0}$ and $f_{\mathrm{N}}$.

All frequencies occurring in the spectrum of RR Lyr can be explained through combinations of the main frequency $f_{0}$, its harmonics $k f_{0}$ and the nonradial mode $f_{\mathrm{N}}$ (see Fig. 3). In this way, Eq. (3) can be rewritten as

$$
\begin{aligned}
f(t)= & A_{0}+\sum_{k=1}^{n}\left[A_{k} \sin \left(2 \pi k f_{0} t+\phi_{k}\right)\right. \\
& +A_{k+} \sin \left(2 \pi\left((k-1) f_{0}+f_{\mathrm{N}}\right) t+\phi_{k+}\right) \\
& \left.+A_{k-} \sin \left(2 \pi\left((k+1) f_{0}-f_{\mathrm{N}}\right) t+\phi_{k-}\right)\right] \\
& +B_{0} \sin \left(2 \pi\left(\left(f_{\mathrm{N}}-f_{0}\right) t+\phi_{\mathrm{B}}\right)\right)
\end{aligned}
$$

In this decomposition, referred to as the "two-frequency model", the Blazhko frequency is the result of a beating between two real frequencies, since $f_{\mathrm{B}}=f_{\mathrm{N}}-f_{0}$. 

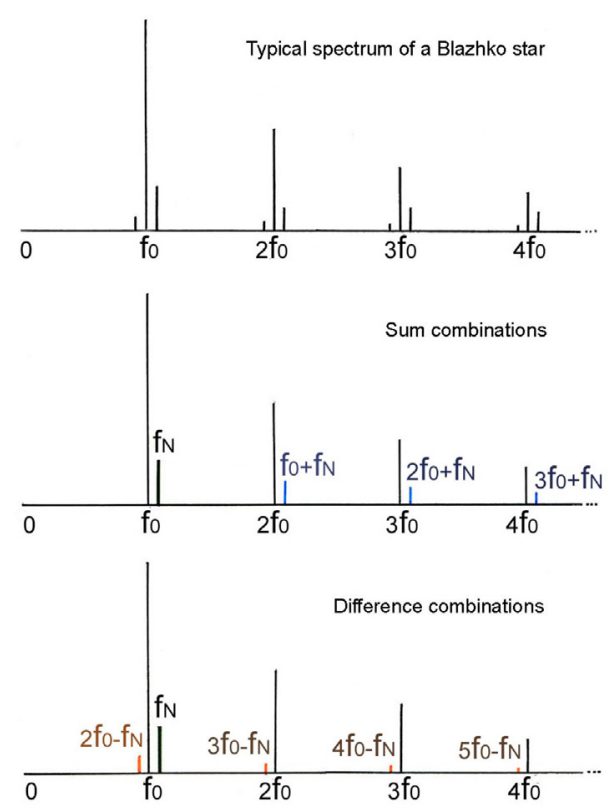

Fig. 3. The typical frequency spectrum of a Blazhko star (top) can be decomposed into combinations $k f_{0} \pm f_{\mathrm{N}}$ with $k=0,1,2, \ldots$ (center: sum combinations, and bottom: difference combinations) of $f_{0}$ and an additional mode with a frequency $f_{\mathrm{N}}$ (right side peak) close to $f_{0}$. A possible explanation for the lower left side peaks is that they result from a combination with a higher order harmonic than the right side peaks.

The two models expressed in Eqs. (3) and (4) are mathematically equivalent (see also Tab. 3). However, if the frequencies observed in the spectrum merely result from linear combinations of $f_{0}$ and $f_{\mathrm{N}}$, the "two-frequency model" may be more accurate. This model explains the (equidistant) triplets in a natural way and may even explain the unequal amplitudes in the triplet structures observed in the majority of the Blazhko stars (see e.g., Alcock et al. 2003). The right side (higher frequency) peak in the triplet at $f_{0}$ corresponds to the mode with frequency $f_{\mathrm{N}}$. The right side peaks in the triplet structures around the harmonics result from the sum combinations, whereas all the left side (lower frequency) peaks can be explained by differences. The left side peaks that are generally lower (and sometimes barely visible) may be explained as the result of a combination with a higher harmonic. This straightforward model raises a lot of complicated questions, e.g., concerning the nature of combination frequencies, their amplitudes and phases, and the possible role of resonances, but it is certainly worth further exploration.

\subsection{A "static" phase interval in the pulsation cycle}

Figure 4 shows the combined RR Lyr $V$ data folded with the main pulsation period $f_{0}=1.7641704 \mathrm{c} / \mathrm{d}$ (top panel), as well as the residuals from the mean light curve (lower panel). The mean light curve is defined by the components of the fit given in Table 3 , varying with only $f_{0}$ and its 10 significant harmonics (up to $11 f_{0}$ ). Figure 4 clearly displays an interval in the pulsation cycle, roughly $\phi=0.72-0.82$, during which the star's intensity barely changes over the Blazhko cycle. The "static" phase interval is right after the bump and shortly before the start of the steep ascending branch, during the infalling motion and between the assumed phases of early and main shock (Gillet \& Crowe 1988). Reduced scatter in the descending branch of Blazhko stars is a common feature. In the folded light curve of RR Lyrae as shown

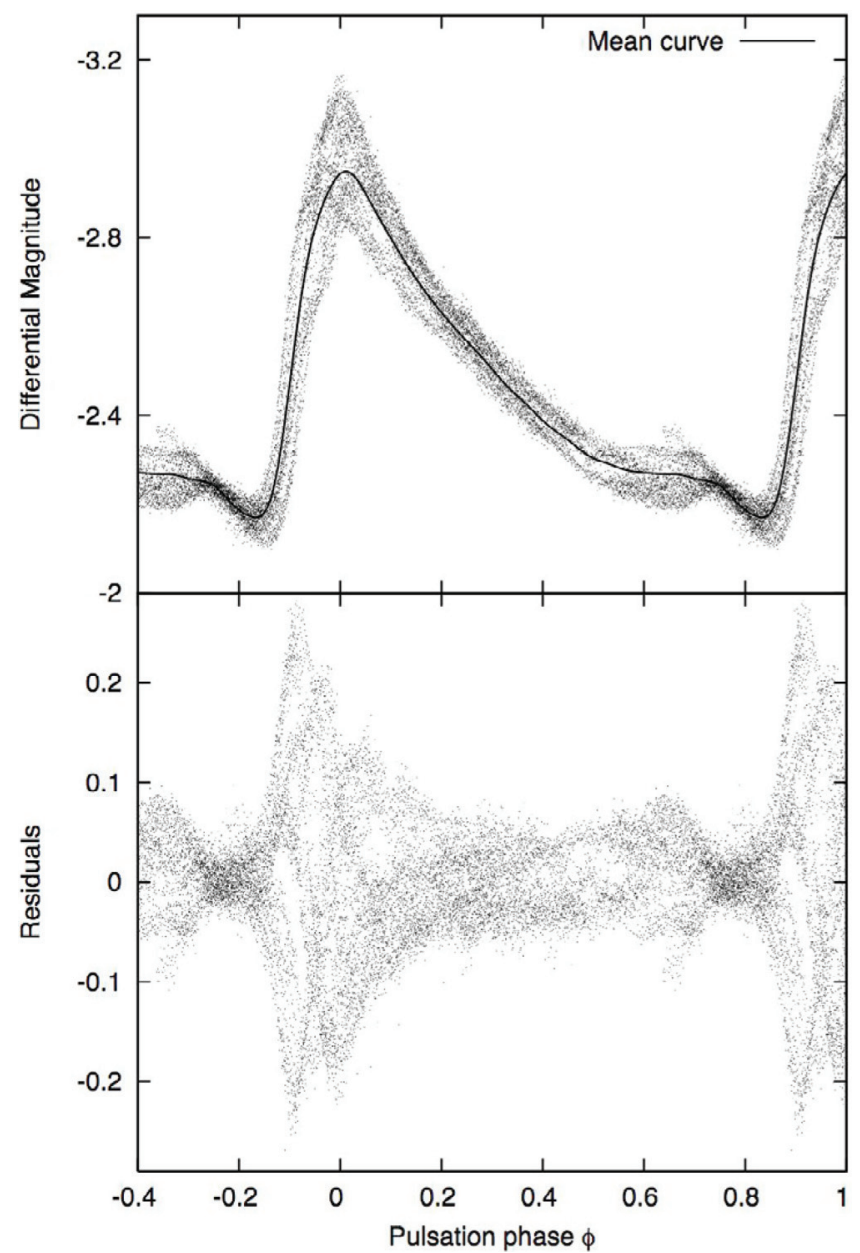

Fig. 4. Top: the combined RR Lyr photometry of 2003-2004 folded with the main pulsation period. The mean light curve derived from all data is displayed as a solid line. Bottom: residuals after subtraction of the mean light curve, clearly showing a phase interval during which the brightness barely changes over the Blazhko cycle.

in Fig. 4, however, the decrease in scatter is very distinct. The folded photographic light curve of RR Lyr from the years 19431945 published by Szeidl \& Kolláth (2000, their Fig. 1) also indicates a slightly reduced scatter in this phase interval. The folded light curve of RR Lyr published by Smith et al. (2003) shows the generally reduced scatter on the descending branch, but the interval of even more reduced scatter shortly before minimum light is not as pronounced. This may be due to the shorter 1996 data set; more data may have resulted in a more unambiguously "static" phase interval. However, other Blazhko stars seem not to show this feature, e.g., the folded light curve of XZ Cyg published by LaCluyzé et al. (2004), or those of some Blazhko stars detected by the MACHO survey (see, e.g., Kurtz et al. 2000, their Fig. 4). Is it still an open question whether this feature is a unique, and coincidental, property of RR Lyr, or if it is also observable in other Blazhko targets.

Nevertheless, this observational fact deserves a closer look; an alternative way of approaching RR Lyrae light curves may also provide new insight into the Blazhko problem.

\subsection{The amplitudes of the modulation components}

From Table 3 it is clear that the right side peaks in the triplet structures have higher amplitudes. The left side peak at $6 f_{0}-f_{\mathrm{B}}$ 
Table 4. Side lobe amplitude ratios $R_{k}$, phase differences $\Delta \phi_{k}$ (in degrees) and asymmetry parameters $Q$ as defined in the text, and their respective errors for the 2004 photometric $V$ data. $k$ denotes the multiplet order.

\begin{tabular}{ccccccc}
\hline \hline$k$ & $R_{k}$ & $\sigma_{R_{k}}$ & $\Delta \phi_{k}$ & $\sigma_{\Delta \phi_{k}}$ & $Q$ & $\sigma_{Q}$ \\
\hline 1 & 5.881 & 0.324 & -12.017 & 9.896 & 0.709 & 0.014 \\
2 & 2.150 & 0.086 & -143.067 & 10.225 & 0.365 & 0.018 \\
3 & 2.306 & 0.090 & -3.703 & 7.231 & 0.395 & 0.016 \\
4 & 2.987 & 0.156 & 207.136 & 7.114 & 0.498 & 0.020 \\
5 & 4.435 & 0.476 & -43.743 & 6.982 & 0.632 & 0.032 \\
6 & 4.656 & 0.868 & -128.733 & 7.070 & 0.646 & 0.054 \\
7 & 2.489 & 0.354 & -60.533 & 9.415 & 0.427 & 0.058 \\
\hline
\end{tabular}

does not have a higher signal-to-noise ratio than 3.5, and for the higher order side lobes (from $8 f_{0}$ onwards) always at least one of the side peak components is below the 3.5-noise level.

As Jurcsik et al. (2005) concluded from their analysis of RR Gem, the amplitudes of the modulation components decrease much less steeply than the amplitudes of the harmonics of the main frequency. This is also the case for RR Lyr (see Table 3).

Table 4 lists the amplitude ratios, phase differences and their errors for the first seven modulation component pairs, defined as

$R_{k}=A_{k f_{0}+f_{\mathrm{B}}} / A_{k f_{0}-f_{\mathrm{B}}}$

and

$\Delta \phi_{k}=\phi_{k f_{0}+f_{\mathrm{B}}}-\phi_{k f_{0}-f_{\mathrm{B}}}$,

where $f_{0}$ is the radial mode frequency and $f_{\mathrm{B}}$ the Blazhko frequency. In Table 4 we also display the so-called asymmetry parameter $\left(Q=\frac{A_{+}-A_{-}}{A_{+}+A_{-}}\right)-$introduced by Alcock et al. (2003) to quantify the degree of asymmetry in the peaks. For all the 731 Blazhko stars detected by the MACHO survey, the asymmetry parameters show a rather broad distribution and peaks at +0.3 , indicating that on average the right side peaks in the triplets have amplitudes almost twice as high as the left side peaks. (Note: $-1.0 /+1.0$ refers to complete asymmetry, 0 to a symmetric triplet.) For the 2004 RR Lyr data, the error-weighted mean value of the asymmetry parameter $Q$ over the first 7 modulation component pairs is about +0.52 . This indicates a 'higherthan-average' asymmetry to the right. In this respect, however, we note that the quantification of this asymmetry is subject to some uncertainty. This was nicely illustrated by Jurcsik et al. (2005), who checked the stability of their results by analyzing different subsets of their extensive data set on the low amplitude Blazhko star RR Gem, and concluded that the phase coverage over the Blazhko cycle affects the amplitudes of the observed side lobes.

For RR Lyr, however, following the studies of Szeidl \& Kollath (2000, and references therein) and Smith et al. (2003), all present photometric data sets of RR Lyr show the higherfrequency side lobes to have higher amplitudes. This consistent picture points to a real difference in the amplitudes of the modulation components.

\subsection{Variations during the Blazhko cycle}

The temporal variation of the light curve shape parameters may provide decisive information about the Blazhko mechanism that can be used to eliminate some models for the Blazhko phenomenon. A powerful tool for analyzing and comparing light curves of pulsating stars is provided by Fourier decomposition, a technique first applied by Simon \& Lee (1981).
Table 5. Log on the subsets of 2004 observations used to construct the light curves at different phase intervals in the Blazhko cycle.

\begin{tabular}{ccc}
\hline \hline$\Delta \Psi$ & HJD $(-2450000)$ & $N$ \\
\hline $0.0-0.1$ & $3176.6256-3253.5479$ & 2943 \\
$0.1-0.2$ & $3177.6033-3334.6154$ & 797 \\
$0.2-0.3$ & $3142.6229-3336.6100$ & 893 \\
$0.3-0.4$ & $3184.8050-3304.5697$ & 933 \\
$0.4-0.5$ & $3188.6489-3308.3469$ & 1533 \\
$0.5-0.6$ & $3154.5992-3312.6633$ & 848 \\
$0.6-0.7$ & $3160.6174-3316.5163$ & 1721 \\
$0.7-0.8$ & $3161.6166-3319.6172$ & 1604 \\
$0.8-0.9$ & $3205.6163-3322.5803$ & 1256 \\
$0.9-1.0$ & $3170.5917-3288.6396$ & 1112 \\
\hline
\end{tabular}

The calculated Fourier parameters that are the amplitude ratios

$R_{k 1}=A_{k} / A_{1}$

and the epoch-independent phase differences

$\phi_{k 1}=\phi_{k}-k \phi_{1}$,

offer a way to quantify the asymmetry of the lightcurves. Nearly sinusoidal light curves (like, e.g., of RRc stars) lead to low amplitude ratios and large phase differences, whereas the opposite holds for highly asymmetric light curves.

Our 2004 data were divided into 10 subsets, corresponding to uniformly distributed phase intervals throughout the Blazhko cycle (e.g., $\psi=0.0-0.1, \psi=0.1-0.2$, etc.), where Blazhko phase $\psi=0$ is defined as the phase of maximum Blazhko amplitude. The minimum and maximum numbers of data in these subsets are 797 and 3082. The time base for each of these intervals, as well as the number of data used to construct the mean light curves, are listed in Table 5. For each of the Blazhko phase intervals, we determined a mean light curve. Figure 5 shows the data for each of the intervals, the mean light curves obtained from these data per Blazhko phase interval, as well as the mean light curve derived from the fit to all data.

When defining mean light curves over a Blazhko phase interval, one has to deal with two sources of uncertainties:

1. For RR Lyr, a Blazhko phase interval with a width of 0.1 corresponds to almost 4 days. At some phases in the Blazhko cycle, especially those around maximum light, the light curves change very rapidly. Hence, we stress that the values of the Fourier parameters given in this paper correspond to mean values per Blazhko phase interval.

2. On the other hand, it is known that the Blazhko effect changes in strength, and that it may not exactly repeat from one cycle to the next, as mentioned in Sect. 3.1. Additional periodicities, as were found by Walraven (1949) and Fringant (1961), could not be disentangled from this data set. The variations may also be caused by nonperiodic changes in the light curve shape. All of these factors represent an additional source of uncertainty in defining the "mean light curves" per Blazhko phase interval. However, our data have been gathered over a rather short time span compared to the "4-year cycle" and the light curves from Blazhko intervals of subsequent cycles overlap quite well.

We have based the error calculations for the Fourier parameters in this paper on the residuals of the fit given by the mean light curve per Blazhko interval. This implies that if the scatter around the mean light curve per Blazhko interval is quite large, which 

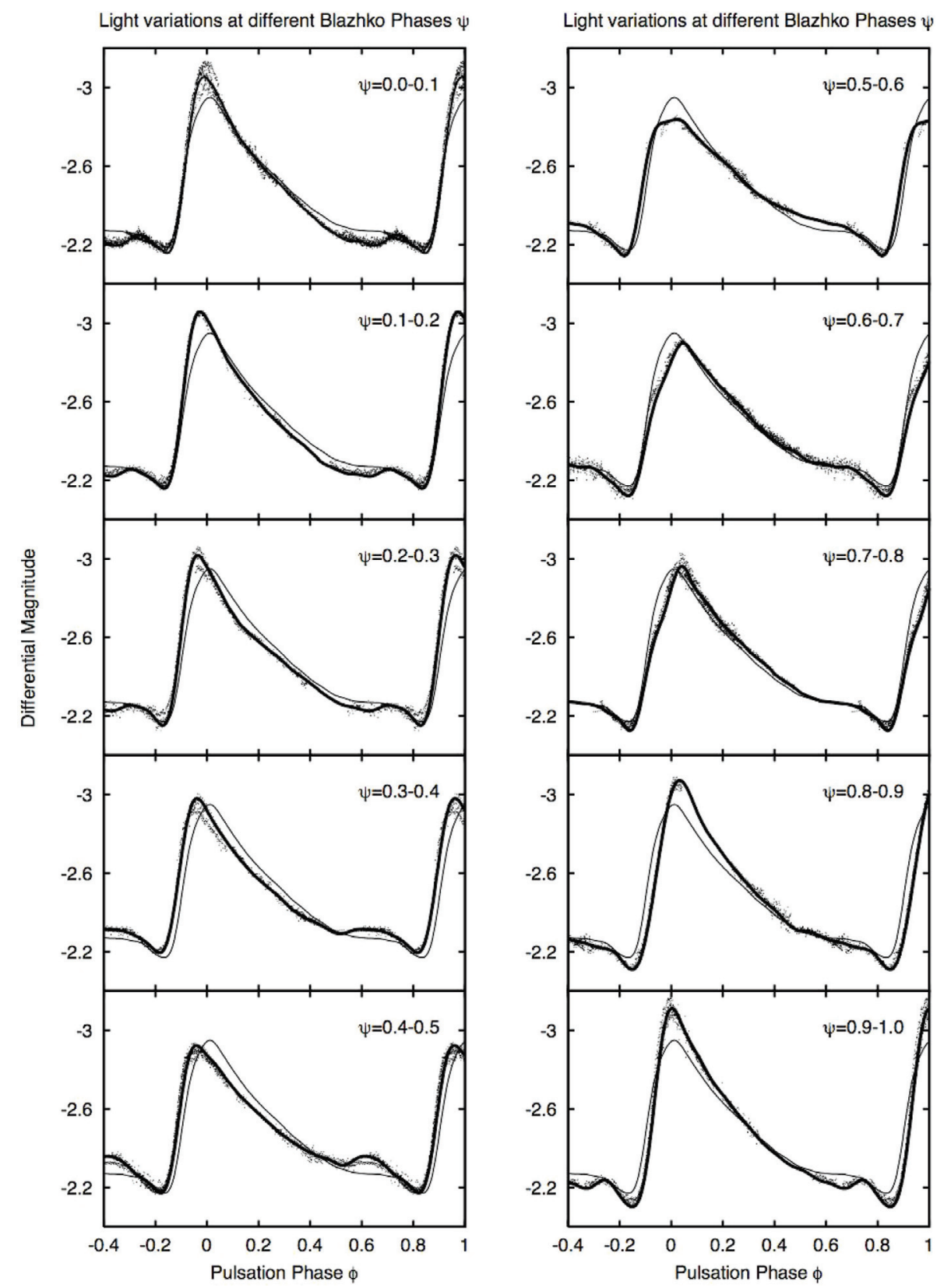

Fig. 5. Pulsation light curves (thick solid lines) constructed from the data (dots) falling into the 0.1 phase intervals of the Blazhko cycle. The mean light curve over all Blazhko phase intervals is also shown as a thin solid line.

is especially the case around maximum Blazhko amplitude (see Fig. 5), the error bars on the mean light curve as well as those on the derived Fourier parameters will become larger. (Note that a higher number of fitted data points also lowers the errors.)

Nevertheless, we call for caution in interpreting these "mean light curves", and stress that the light curves, and their characteristics shown in Figs. 5-7, are representative for this data set. We cannot exclude that there is an influence of as yet unknown periodicities on the mean light curves. For data gathered at another time, the picture may change. Therefore, it would be interesting to follow up on RR Lyr and the changes in its Blazhko behavior.

A first inspection of the mean light curves at different Blazhko phases reveals that RR Lyr shows both amplitude and phase modulation. The ascending branch moves back and forth in the course of the Blazhko cycle. Figure 6 shows the pulsation phases of maximum and minimum light, as a function of the Blazhko phase, as well as the difference in phase between minimum and maximum light. Balázs \& Detre (1943) found that the time interval between minimum and maximum varies between 0.067 (at $\psi=0.90$ ) and 0.121 (at $\psi=0.09$ ). We find larger values ranging from $0.14( \pm 0.02)$ to $0.21( \pm 0.02)$, with the lower values during the first half of the Blazhko cycle and a steep increase around the minimum of the Blazhko amplitude. This observation illustrates that the light curves observed by Balász \& Detre (1943) had branches ascending more steeply than the ones we observed. There is no strict repetition of the light curve shape from one Blazhko cycle to the next, as already shown from our data set spanning 10 Blazhko cycles. Additionally, the so-called "4-year cycle" (Detre \& Szeidl 1973) known from the literature, is connected with stronger and weaker phases of the Blazhko effect.

In the mean light curves (see Fig. 5), the so-called bump, the slight increase in the brightness at a pulsation phase $\phi \simeq 0.7$, gets more pronounced at certain Blazhko phases, e.g., $\psi=0.4-0.5$. 


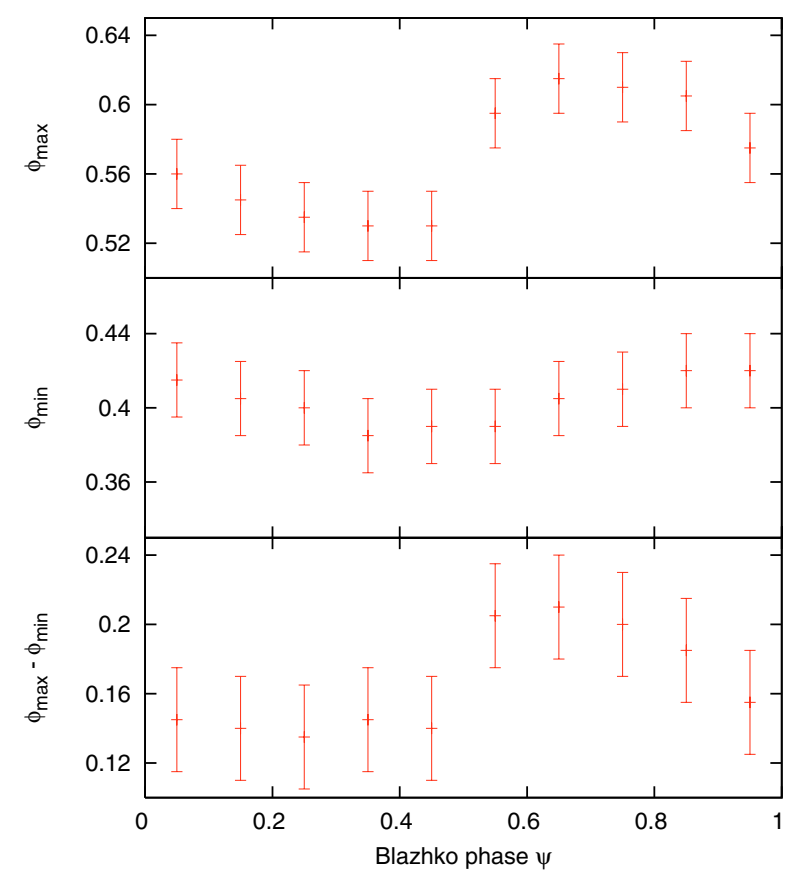

Fig. 6. Pulsation phase of maximum light (top), of minimum light (center), and pulsation phase interval between minimum and maximum light (bottom) as a function of the phase in the Blazhko cycle.

It also seems that the bump occurs later closer to the phase of Blazhko maximum. A clear hump (a slight hesitation in the course of the light increase at $\phi \simeq 0.9$ ) can be seen in the Blazhko phase interval $\psi=0.6-0.8$. The bump is usually associated with the early shock in the atmospheres of RR Lyrae stars, and the hump with the main shock, though there is no consensus on the exact relationships between the shock waves and the luminosity variations yet (see, e.g., Gillet \& Crowe 1988; Carney et al. 1992).

A higher-order harmonic fit was made to the mean light curve defined for each of the subsets, according to:

$f(t)=A_{0}+\sum_{k=1}^{8} A_{k} \sin \left(2 \pi k f_{k} t+\phi_{k}\right)$.

The changes in the light curve shape are most pronounced in the height of maximum which itself varies strongly. These variations occur so quickly that for some 0.1 -phase intervals the portions of light curves observed within an interval of some days already show a rather large scatter. Hence the fit to the curve yields mean values with larger error bars for the Fourier parameters. The course of the Fourier parameters over the Blazhko cycle is given in Figure 7 . Note that the maximum in the amplitude ratios does not occur at the highest Blazhko amplitude $\psi=0$, but rather in the phase interval $\psi=0.4-0.5$. Indeed, the amplitudes $A_{1}$ decrease more steeply towards the Blazhko minimum than the amplitudes of the harmonics of the main frequency. Our observed Fourier parameters fall within the range of theoretical values calculated by Dorfi \& Feuchtinger (1999) and Feuchtinger (1999). Fitting the observed changes in the Fourier parameters with subsequent hydrodynamical models for a given period would be a challenging and informative confrontation between theoretical work and observations. Finally, we note that the value of $[\mathrm{Fe} / \mathrm{He}]$ calculated from the period and the Fourier parameter $\phi_{31}$ close to its lowest value around Blazhko maximum phase (4.99 in the sine frame, 1.85 in the cosine frame - see
Fourier parameters over the Blazhko cycle

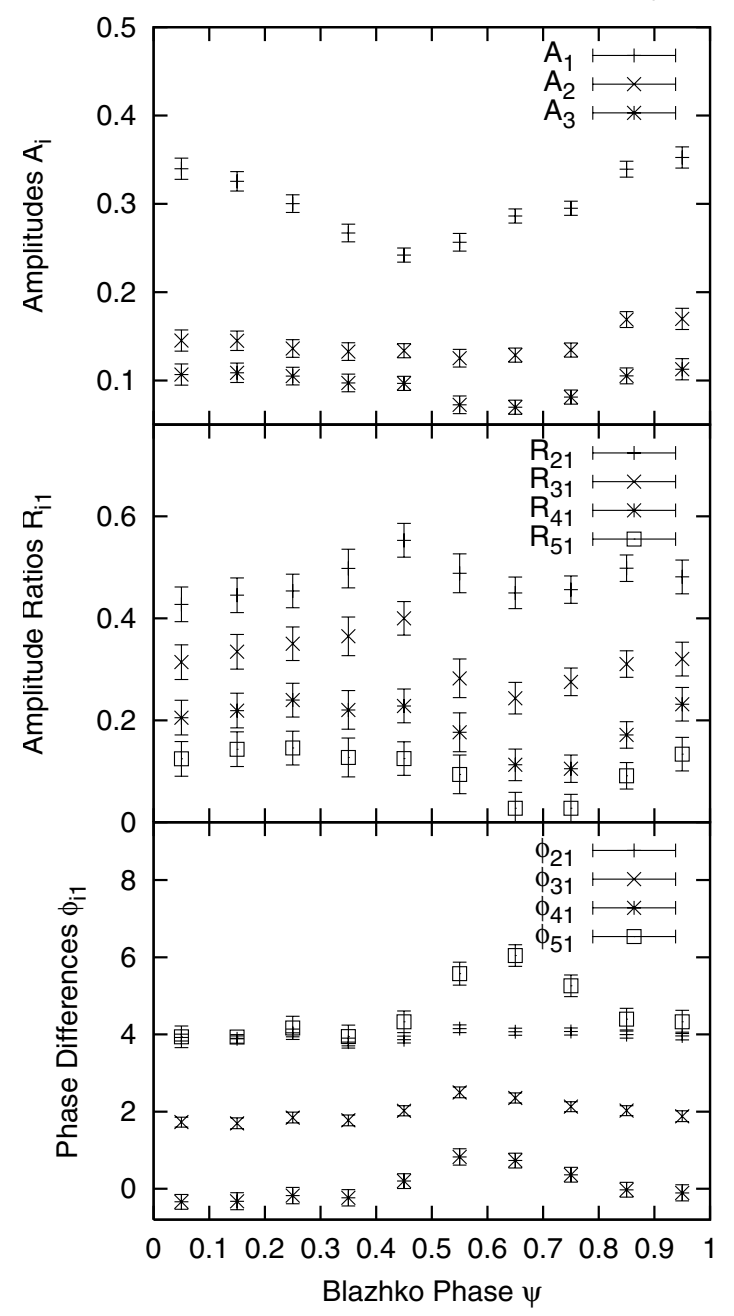

Fig. 7. Fourier parameters of the $V$ light curve in different phases of the Blazhko cycle. Upper panel: amplitudes in mag. Middle panel: amplitude ratios as defined in the text. Lower panel: epoch-independent phase differences in radians and with respect to a cosine frame, to allow comparsion with the theoretical Fourier parameters calculated by Feuchtinger (1999).

Fig. 7) with the formula given by Kovács \& Jurcsik (1996) yields the known $[\mathrm{Fe} / \mathrm{H}]=-1.39$ of RR Lyr (Beers et al. 2000).

We calculated mean brightnesses and intensities for each of the phase intervals. Unfortunately, the accuracy of this data set did not allow us to detect a significant variation in the mean brightness or intensity of RR Lyr over the Blazhko cycle. This implies that the mean brightness variations do not exceed 0.02 mag. From a survey on 731 Blazhko variables, Alcock et al. (2003) conclude that there is a variation with the Blazhko period having an overal amplitude of about 0.006 mag in the average brightness of the stars.

\section{Discussion}

\subsection{Confrontation with the models for the Blazhko effect}

In their present form, neither of the two models discussed in Sect. 1.1 satisfactorily explains the variety of features diplayed by Blazhko stars, though some of these peculiarities have been partially accounted for. For instance, Van Hoolst et al. (1998) 
predict a higher probablity of excitation of nonradial modes for RRab stars than for RRc stars, though not in the degree observed. A few other examples of still insufficiently resolved characteristics are the asymmetry of the side peaks, and the tendency of the right side peak in the triplets to be higher, both of which were confirmed by our observations of RR Lyr. Models should also explain why some Blazhko stars show pure amplitude modulation, some pure phase modulation, and others both types of modulation. RR Lyrae clearly shows both.

Note that quintuplet structures in the frequency spectra of RR Lyrae stars have never been observed convincingly enough to conclude their reality (Alcock et al. 2000, 2003; Moskalik \& Poretti 2003). In line with the previous studies, the present data set reveals no significant quintuplet frequencies.

It is also clear from this data set that the amplitudes of the modulation components in the frequency spectrum decrease much less steeply than the amplitudes of the harmonics of the main radial frequency. This observation was already made by Jurcsik et al. (2005) in their analysis of photometric data of the Blazhko star RR Gem. Any valid model for the Blazhko effect should also account for this observation.

The identification of the nonradial modes in modulated RR Lyrae stars is essential for narrowing down the possible explanations for the Blazhko effect. High-resolution spectroscopic data are the best diagnostic for finding and identifying nonradial modes in pulsating stars. Earlier results obtained from spectroscopic data (Kolenberg et al. 2003) pointed towards the presence of a nonradial mode with low degree, in agreement with the models, but not discriminating among them. A more precise identification was hampered by the limited signal-to-noise ratio of the data and the incomplete sampling over the Blazhko cycle. A combined photometric and spectroscopic campaign carried out over several Blazhko cycles is the best way to observationally assess the Blazhko problem.

\subsection{Features in the light curves over the Blazhko cycle}

Our observation of a "static" phase interval in the pulsation cycle (Sect. 3.3), during which the star's intensity barely changes over the Blazhko cycle, may shed new light upon approaches to explain the Blazhko effect. It occurs between the phases of early and main shock in the star, associated with respectively the bump and the hump. It would be interesting to investigate whether other Blazhko stars show a similar feature.

Furthermore, the obvious changes in the strength and position of the bump over the Blazhko cycle, observed in RR Lyr and other Blazhko stars, deserve a more profound study. Depending on its origin, still a matter of controversy, the bump can provide a clue about the star's interior structure or its pulsational behavior. Two competing models have been proposed to explain the bump. For a more detailed description of the "infall hypothesis" and the 'echo model', we refer to Gillet \& Crowe (1988) and Carney et al. (1992).

From our data set, there is no clear indication of any change in the Blazhko phase between the data recorded in 2003 and those gathered in 2004, nor any strong indication for the end of a longer cycle during our 2004 observations. It would be interesting to find out whether the "4-year cycle" of RR Lyr is still as prominent as it was over the past half century, and how well it really describes the long-term behavior of RR Lyr. Future photometric data of RR Lyr can serve this purpose.

\subsection{The changing Blazhko period}

Not only the amplitude but also the length of the secondary period seems to be variable. As was reported by Szeidl \& Kolláth (2000), RR Lyr's primary period has shown small increases and decreases since its discovery in 1901 . No regularity can be found in the changes of the primary period. An important result following from the present data set on RR Lyr is that the Blazhko period, previously known to be about 40.8 days, has become notably shorter. Our analysis points towards a Blazhko period of about 39 days. A shorter Blazhko period for RR Lyr was already pointed out by Belserene (1999) who obtained 161 differential $B$ measurements over a total time span of 477 days in 1993 and 1994. She finds a prominent secondary peak in the data at 1.7897 $\mathrm{c} / \mathrm{d}$, corresponding to our $f_{0}+f_{\mathrm{B}}$ or $f_{\mathrm{N}}$. Her best frequencies for the main pulsation and the side peak frequency point towards a Blazhko period of 39.2 days, though the error bars are larger than the ones derived from the present data set. Smith et al. (2003) analyzed 2573 differential $B$ and 2461 differential $V$ data spanning a 77-day interval in 1996. From their fits to the data they find a value of $40 \pm 1$ day for the Blazhko period, adding weight to the observations made by Belserene (1999). Our much denser data set spans 10 consecutive Blazhko cycles, and hence leads to smaller error bars on the frequencies. We can now safely state that the Blazhko period is about 39 days, shorter than the previously mentioned value of 40.8 days. Belserene's (1999) data indicate that already in 1993-1994 the Blazhko period appeared to be shorter.

The changing Blazhko period of RR Lyr reminds one of the recent results published by LaCluyzé et al. (2004) on the changing Blazhko period of XZ Cyg. During the first half of the 20th century, XZ Cyg had a Blazhko period of about 57.4 days. Starting in 1965 , the primary period underwent a steep decline (in several steps), accompanied by an increase in the Blazhko period to 58.5 days. Extensive analyses of old and new photometry of XZ Dra (Jurcsik et al. 2002b) and RV UMa (Kanyo 1976; Kovacs 1995) confirmed that these stars also show coincident changes in their Blazhko period and primary period. These changes have different signs and orders of magnitude. Any theoretical explanation that requires the Blazhko period to be exactly equal or directly proportional to the rotation period of the star would imply changes in the star's rotation rate.

Throughout the literature on RR Lyr, there has always been mention of a Blazhko period of about 41 days. Prager (1916) was the first to find the secondary oscillation in the phases of RR Lyr's light maxima from data by Wendell $(1909,1914)$. Shapley (1916) investigated the changes in spectrum, period, and light curve of RR Lyr, and obtained a secondary period of 40 days. Struve \& Blaauw (1948) found the radial velocity curve to oscillate with a period of approximately 75 pulsation cycles, or about 42 days. Walraven (1949) published the first photoelectric observations of RR Lyr, also performed in 1947, mentioning a 41-day cycle. Fringant (1961) describes RR Lyr's secondary cycle as the mean value of 72 primary cycles, resulting in a period of 40.8 days. The important study by Preston et al. (1965) makes notice of a modulation period of 41 days, and adopts Fringant's (1961) published value. Table 6 shows a summary of the Blazhko periods of RR Lyr quoted in previous papers devoted to the star. Unfortunately, error bars on the Blazhko period are rarely given. Note that this table only contains a listing of some of the main published data sets of RR Lyr. An exhaustive analysis of all still available previous data sets is beyond the scope of this paper. Nevertheless, we think this concise overview is important to show that the Blazhko period indeed has become shorter. 
Table 6. Published Blazhko periods from some previous data sets of RR Lyr. If error bars are given in the publication, they are added. We stress that this table is far from exhaustive, but just indicative of the likely change of the star's Blazhko period.

\begin{tabular}{lcccc}
\hline \hline Reference & Years & $\Delta T(\mathrm{~d})$ & $P_{\mathrm{B}}$ (listed) & Comments \\
\hline Shapley (1916) & $1899-1914^{(1)}$ & 5540 & 40 & $P_{\mathrm{B}} \simeq 70 P_{0}^{(2)}$ \\
Balász \& Detre (1943) & $1935-1941$ & 2188 & 41 & $P_{\mathrm{B}} \simeq 72.4 P_{0}^{(3)}$ \\
Struve \& Blaauw (1948) & 1947 & 42 & 42 & $P_{\mathrm{B}} \simeq 75 P_{0}^{(4)}$ \\
Walraven (1949) & 1947 & 201 & $41^{(5)}$ & \\
Fringant (1961) & $1949-1956$ & $2500+$ & $40.8^{(1),(6)}$ & $P_{\mathrm{B}} \simeq 72 P_{0}$ \\
Preston et al. (1965) & $1961-1964$ & $1026(\mathrm{Ph}),. 855(\mathrm{Sp} .)^{(7)}$ & $40.8^{(8)}$ & \\
Detre \& Szeidl (1973) & $1899-1972$ & $25000+$ & $41^{(9)}$ & \\
Belserene (1999) & $1993-1994$ & 477 & 39.2 & \\
Smith et al. (2003) & 1996 & 77 & $40( \pm 1)$ & \\
This paper & $2003-2004$ & 421 & $38.8 \pm 0.1$ & \\
\hline
\end{tabular}

(1) Result also based on older data sets by other observers.

(2) Derived from the variations in time of the median magnitude. Also mention of a longer cycle of 16.5 years.

(3) The authors write that the light curve variations of RR Lyr can most easily be discribed by a superposition of two pulsations with the period $P_{0}=0.567 \mathrm{~d}$ and $P_{1}=0.559 \mathrm{~d}$.

(4) From the variation in the radial velocity curve.

(5) An additional period is mentioned, about three times longer than $P_{\mathrm{B}}$.

(6) The author mentions four periods of RR Lyr: $P_{0}=0.5668 \mathrm{~d}, P_{\mathrm{B}}=40.8 \mathrm{~d}, P_{3} \simeq 3 P_{\mathrm{B}}$, and $P_{4}$ of about 10 years.

(7) Simultaneous spectroscopic and photometric data.

(8) Reference to Fringant's (1961) elements.

(9) First mention of RR Lyr's "4-year cycle". The authors report that during one and the same "4-year cycle" $P_{\mathrm{B}}$ remained constant.

It would be interesting to know when the change in the Blazhko period began, and whether it took place abruptly or rather continuously. As mentioned above, data gathered in 1993 (Belserene 1999) and 1996 (Smith et al. 2003) already indicated a shorter Blazhko period.

In the cases of XZ Cyg, XZ Dra and RV UMa, the change in the Blazhko period was associated with a change in the fundamental period. As with many RR Lyrae stars, the main period of RR Lyr has been subject to strong variation, in which no regularity can be noticed. The $\mathrm{O}-\mathrm{C}$ diagram for the primary period of RR Lyr shown in the review by Szeidl \& Kollath (2000) (their Fig. 3) spanning a century of observations (1899-1999) presents a series of ups and downs. The data from Struve \& Blaauw (1948) and Walraven (1949) have been taken when the O-C diagram shows a steep decline. The same is true for the data published by Fringant (1961), which were gathered between 1947 and 1959. The O-C diagrams published by Balász-Detre \& Detre (1962) and by Szeidl (1976, his Fig. 7) display that the changes in both the pulsation period and Blazhko period in RR Lyr were at times parallel, at other times antiparallel, which makes it hard to draw firm conclusions about the relation between the period changes in RR Lyr. The constancy or variability of the primary period is connected with the interesting but still unanswered question of what initiated the change in the Blazhko period.

From our data set no further significant frequencies could be detected. However, as Fig. 8 shows, the residuals from the fit still show scatter, especially around the phase of light maximum. The enhanced scatter around maximum light may be in part a consequence of the difficulties of fitting a steep function (the rising branch) with a Fourier series. On the other hand, the scatter in the residuals could also indicate the presence of periodic or nonperiodic changes in the light curve. But since the mean residual value $(0.021 \mathrm{mag})$ is of the order of the noise level in the data set, we can draw no conclusions about its cause. Additional third and fourth frequencies in the light curve of RR Lyr were identified by Walraven (1949) and Fringant (1961), but the long-term existence of these cycles has not yet been established. Therefore,

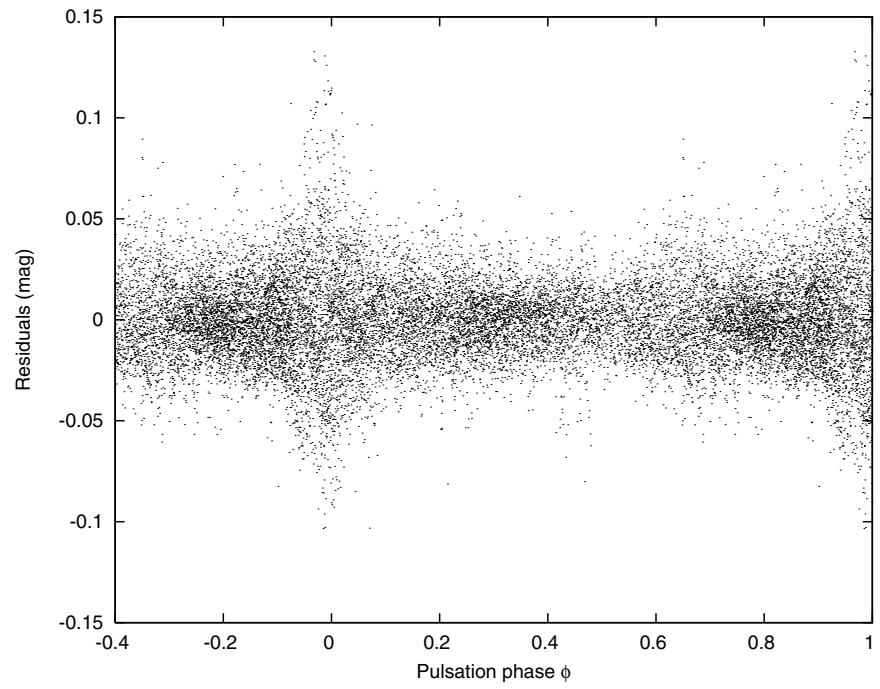

Fig. 8. Residuals after subtraction of the fit to the data described in Table 3. There is an increased scatter around the phase of maximum light.

follow-up campaigns devoted to RR Lyr are of great scientific importance.

\section{Conclusions}

In this paper we present the results of a frequency analysis of an extensive set of photometric data of the Blazhko star RR Lyr, which was obtained with six different telescopes and over a total time span of more than 10 Blazhko cycles in 2003 and 2004. The main results can be summarized as follows.

- The light curves spanning over 10 Blazhko cycles can be satisfactorily represented by a Fourier sum with frequencies of the form of $k f_{0}, k f_{0} \pm f_{\mathrm{B}}$, with $k=0,1, \ldots, 11$, $f_{0}=1.7641704 \pm 0.0000048 \mathrm{c} / \mathrm{d}$, and $f_{\mathrm{B}} \simeq 0.02575 \mathrm{c} / \mathrm{d}$. An alternative representation was proposed in Sect. 2.2 of 
this paper, decomposing the variation in terms of combinations of the main frequency $f_{0}$, its harmonics and one additional nonradial mode connected to the frequency $f_{\mathrm{N}}=$ $1.7899187 \pm 0.000026 \mathrm{c} / \mathrm{d}$, which has smaller amplitude and behaves in a linear fashion.

- However, there is some residual scatter after subtraction of the best fit obtained for the data. From this data set, we were not able to disentangle additional frequencies.

- The new data reveal that the Blazhko period, previously known to be about 40.8 days, has become remarkably shorter with a value of about 38.8 days. Similar observations have been made for other Blazhko stars, constraining the possible explanations for the Blazhko effect.

- Moreover, our data show that there is a phase interval $(\psi=$ $0.72-0.82$ ) in the pulsation cycle, right after the phase of the so-called bump, during which the star's intensity barely changes over the Blazhko cycle.

- As in the majority of amplitude and phase modulated RRab stars, and as shown by previous studies of the target, the triplet structures observed in the frequency spectrum of RR Lyr show a strong asymmetry to the right. This still puzzling statistical fact may be explained by the simple Fourier decomposition into combination frequencies proposed in Sect. 3.2, in which the left side peaks within the same triplet result from a combination with a higher order harmonic than the right side peaks and hence have a smaller amplitude. The simple picture may be disturbed by resonances in the star, which could enhance or diminish the actually observed amplitudes of the frequencies.

- A study of the variations of the light curve shape over the Blazhko cycle reveals that the strong amplitude and phase modulation in RR Lyr is accompanied by changes in the shape (and position) of the bump, and that the hump is only observed at certain Blazhko phases. Fourier parameters prove very useful to quantize the degree of asymmetry of the light curves. Data covering several complete Blazhko cycles would be needed to find out if such changes in the light curve shape repeat similarly from one Blazhko cycle to the next.

The data set described and analyzed in this paper was obtained in the framework of a combined photometric and spectroscopic campaign devoted to RR Lyrae (see Sect. 1.2). Such combined campaigns, yielding long-term accurate photometry as well as time-resolved, high-signal-to-noise, high-resolution spectroscopy, carried out over several Blazhko cycles are the best way to observationally assess the Blazhko problem.

Acknowledgements. Part of this investigation has been supported by the Austrian Fonds zur Förderung der wissenschaftlichen Forschung, project number P17097-N02. HAS thanks the US National Science Foundation for support under grant AST-0205813. This research has made use of the SIMBAD database, operated at CDS Strasbourg, France. KK is grateful to Bela Szeidl, Johanna Jurcsik and Geza Kovács for fruitful discussions. We thank the referee for useful comments and suggestions.

\section{References}

Alcock, C., Allsman, R., Alves, D. R., et al. 2000, ApJ, 542, 257

Alcock, C., Alves, D. R., Becker, A., et al. 2003, ApJ, 589, 597

Balász, J., \& Detre, L. 1943, Communications of the Konkoly Observatory, No. 18,125
Balázs-Detre, J., \& Detre, L. 1962, Veroeffentlichungen der Remeis-Sternwarte zu Bamberg, 27, 90

Beers, T. C., Chiba, M., Yoshii, Y., et al. 2000, AJ, 119, 2866

Belserene, E. P. 1999, in Anni Mirabiles: A Symposium Celebrating the 90th Birthday of Dorrit Hoffleit, ed. A. G. Davis Philip, W. F. Van Altena, \& A. R. Upgren, Schenectady: L. David Press, 85

Blazhko, S. N. 1907, Astron. Nachr., 173, 325

Breger, M., Stich, J., Garrido, R., et al. 1993, A\&A, 271, 482

Breger, M., Handler, G., Garrido, R., et al. 1999, A\&A, 349, 225

Borkowski, K. J. 1980, Acta Astronomica, 30, 393

Carney, B. W., Storm, J., Trammell, S. R., \& Jones, R. V. 1992, PASP, 104, 44

Castelaz, M. W., Persinger, T., Stein, J. W., Prosser, J., \& Powell, H. D. 1991, Astron. J., 102, 2103

Chadid, M., \& Gillet, D. 1997, A\&A, 319, 154

Chadid, M., Kolenberg, K., Aerts, C., \& Gillet, D. 1999, A\&A, 352, 201

Chadid, M., Wade, G. A., Shorlin, S. L. S., \& Landstreet, J. D. 2004, A\&A, 413, 1087

Cousens, A. 1983, MNRAS, 203, 1171

Detre, L., \& Szeidl, B. 1973, in Variable Stars in Globular Clusters and Related Systems, ed. J. D. Fernie (D. Reidel Publishers), 31

Dorfi, E. A., \& Feuchtinger, M. U. 1999, A\&A, 348, 815

Feuchtinger, M. U. 1999, A\&A, 351, 103

Fringant, A.-M. 1961, Journal des Observateurs, 44, 165

Gillet, D., \& Crowe, R. A. 1988, A\&A, 199, 242

Jerzykiewicz, M., Handler, G., Shobbrook, R. R., et al. 2005, MNRAS, 360, 619

Handler, G., Arentoft, T., Shobbrook, R. R., et al. 2000, MNRAS, 318, 511

Jurcsik, J., \& Kovács, G. 1996, A\&A, 312, 111

Jurcsik, J., Benko, J. M., \& Szeidl, B. 2002a, A\&A, 390, 133

Jurcsik, J., Benko, J. M., \& Szeidl, B. 2002b, A\&A, 396, 539

Jurcsik, J., Sodor, A., Varadi, M., et al. 2005, A\&A, 430, 1049

Kanyo, S. 1976, Comm. Konkoly Observatory Budapest, 69, 1

Kolenberg, K., Aerts, C., Fokin, A., et al. 2003a, ASP Conf. Ser., 292, 171

Kolenberg, K. 2005, ASP Conf. Ser., 335, 95

Kovács, G. 1995, A\&A, 295, 693

Kovács, G. 2001, in Stellar pulsation - nonlinear studies, ed. M. Takeuti \& D. D. Sasselov, Astrophysics and space science library (Dordrecht: Kluwer Academic Publishers), 257, 61

Kovács, G. 2005, A\&A, 438, 227

Kurtz, D. W. 1982, MNRAS, 200, 807

Kurtz, D. W., Alcock, C., Allsman, R. A., et al. 2000, ASP Conf. Ser. 203, ed. L. Szabados, \& D. W. Kurtz, 291

LaCluyzé, A., Smith, H. A., Gill, E.-M., et al. 2004, AJ, 430, 1049

Lenz, P., \& Breger, M. 2005, Comm. in Asteroseismology, 146, 53

Montgomery, M. H., \& O’Donoghue, D. 1999, Delta Scuti Newslett., No. 13

Moskalik, P., \& Poretti, E. 2003, A\&A, 398, 213

Nowakowski, R. M., \& Dziembowski, W. A. 2001, Acta Astron., 51, 5

Prager, R. 1916, Sitzb. Ak. Berlin, 8, 216

Preston, G. W., Smak, J., \& Paczynski, B. 1965, ApJS, 12, 99

Romanov, Yu. S., Udovichenko, S. N., \& Frolov, M. S. 1994, Bul. Spec. Astrophys. Obs., 38, 169

Shapley, H. 1916, ApJ, 43, 217

Shibahashi, H., \& Takata, M. 1995, ASP Conf. Ser., 83, 42

Smith, H. A., Barnett, M., Silbermann, N. A., \& Gay, P. 1999, AJ, 118, 572

Smith, H. A., Church, J. A., Fournier, J., et al. 2003, PASP, 115, 43

Simon, N. R., \& Lee, A. S. 1981, ApJ, 248, 291

Struve, O., Blaauw, A. 1948, ApJ, 108, 60

Szeidl, B. 1976, in Multiple Periodic Variable Stars, ed. W. S. Fitch (D. Reidel Publishers, ASSL, 60, 133)

Szeidl, B. 1988, in Multimode Stellar Pulsations, ed. G. Kovacs, L. Szabados \& B. Szeidl, Budapest: Konkoly Observatory, Kultura, 45

Szeidl, B., Guinan, E. F., Olah, K., \& Szabados, L. 1997, Communications of the Konkoly Observatory, No. 99

Szeidl, B., \& Kolláth, Z. 2000, ASP Conf. Ser., 203, 281

Teays, T. J. 1993, in New Perspectives on Stellar Pulsations and pulsating variable stars, ed. Nemec \& Matthews, Proc. IAU Coll., 139, 410

Van Hoolst, T., Dziembowski, W. A., \& Kawaler, S. D. 1998, MNRAS, 297, 536

Walraven, T. 1949, Bull. Astron. Inst. Netherlands, 11, 17

Wendell, O. C. 1909, Harvard Annals, 69, Part I, 45

Wendell, O. C. 1914, Harvard Annals, 69, Part II, 124 